\title{
Geç-term ve post-term gebeliklerin fetal Doppler akım özelliklerinin karşılaştırılması
}

\section{Comparison of fetal Doppler blood flow characteristics of late-term and post-term pregnancies}

\author{
Muhammed Sıraç Güneş ${ }^{1}$ Süleyman Cemil Oğlak ${ }^{2}$ (D) \\ ${ }^{1}$ Siirt Eğitim ve Araştırma Hastanesi, Kadın Hastalıkları ve Doğum Kliniği, Siirt, Türkiye \\ ${ }^{2}$ Sağlık Bilimleri Üniversitesi, Diyarbakır Gazi Yaşargil Eğitim ve Araştırma Hastanesi, Kadın \\ Hastalıkları ve Doğum Kliniği, Diyarbakır, Türkiye
}

Öz

Amaç: Çalışmanın amacı, 41 hafta ve üzeri komplike olmayan geç-term ve post-term gebeliklerde fetal Doppler akım özelliklerini karşılaştırmak ve bunların doğum sonrası sonuçlara etkisini değerlendirmektir.

Gereç ve Yöntem: Ümraniye Eğitim ve Araştırma Hastanesi Kadın Hastalıkları ve Doğum Polikliniği'ne başvuran düşük risk grubundaki toplam 98 geç-term ve post-term tekiz gebe çalışma grubuna alındı. Tüm gebelerin gebelik haftası, son adet tarihi ve erken dönem ultrasonografi verileri ile teyit edilerek hesaplandı. Hastalara sonografik değerlendirme ile tahmini fetal kilo, amniyotik sıvı hacmi, biyofizik profil ve Doppler (fetal umbilikal arter, orta serebral arter ve maternal uterin arter) incelemeleri yapıldı. Orta serebral ve umbilikal arterlerde pulsatilite ve rezistans indeksleri ile serebroplasental oran Doppler ultrasonografi ile günlük olarak değerlendirildi. Bu gebelerin doğum şekli, yenidoğan doğum kilosu, doğumda mekonyumlu amniotik sıvı varlığı, NST'de fetal distres bulgusu varlığı, yenidoğan 5. dakika Apgar skoru, fetal distres varlığında kordon kan gazı pH değeri ve yenidoğan yoğun bakım ünitesi intiyacı olup olmadığı kayıt altına alındı.

Bulgular: Her iki grubun ortalama uterin arter ve ortalama umbilikal arter pulsatilite indeksleri ile orta serebral arter rezistans indeksi değerleri arasında fark gösterilememiştir. Post-term grupta ortalama umbilikal arter rezistans indeksi ve orta serebral arter pulsatilite indeksi değerleri anlamlı olarak daha düşük bulunmuştur. Geç-term grupta serebroplasental oran daha düşük olsa da bu farklılık istatistiksel olarak anlamlı değildir. Her iki grupta, bebeklerin yeni doğan yoğun bakım ünitesine yatma oranları arasında anlamlı bir fark saptanmadı.

Sonuç: Anormal ve normal serebroplasental oran sonucuna göre yenidoğanın iyilik hali ve yoğun bakım ünitesinde yatma ihtiyacı açısından anlamlı ilişki saptanmamasına rağmen daha geniş serili çalışma gruplarına intiyaç olduğu düşünülmektedir.

Anahtar Sözcükler: Serebroplasental oran, orta serebral arter, umbilikal arter.

\begin{abstract}
Aim: The aim of this study was to compare fetal Doppler flow characteristics in uncomplicated lateterm and post-term pregnancies over 41 weeks and to evaluate their effect on postpartum outcomes.

Materials and Methods: A total of 98 late-term and post-term singleton pregnant women in the low risk group who were admitted to the Umraniye Education and Research Hospital Obstetrics and Gynecology Polyclinic were included in the study group. The gestational week of all pregnant women was calculated by confirming their last menstrual period and early period ultrasonography data. Estimated fetal weight, amniotic fluid index, biophysical profile and Doppler examinations (fetal umbilical artery, middle cerebral artery and maternal uterine artery) were performed by sonographic evaluation.
\end{abstract}

\footnotetext{
Sorumlu yazar: Süleyman Cemil Oğlak

Sağlık Bilimleri Üniversitesi, Diyarbakır Gazi Yaşargil Eğitim

ve Araştırma Hastanesi, Kadın Hastalıkları ve Doğum Kliniği,

Diyarbakır, Türkiye

E-posta: sampson_21@hotmail.com

Başvuru tarihi: 18.09.2019 Kabul tarihi: 28.03.2020
} 
The pulsatility and resistance indices of the middle cerebral and umbilical arteries and cerebroplacental ratio were evaluated daily by Doppler ultrasonography. Delivery type, birth weight, amniotic fluid with meconium at birth, presence of fetal distress finding in NST, 5th minute Apgar score, cord blood $\mathrm{pH}$ value in presence of fetal distress and neonatal intensive care requirement of newborns were recorded.

Results: There was no difference between the mean values of uterine artery pulsatility index, umbilical artery pulsatility index and middle cerebral artery resistance index in both groups. In the postterm group, the mean umbilical artery resistance index and middle cerebral artery pulsatility index values were significantly lower. Although cerebroplacental ratio was lower in the late term group, this difference was not statistically significant. In both groups, no significant difference was found between the rate of hospitalization in the intensive care unit of newborns.

Conclusion: Although there is no significant relationship between abnormal and normal cerebroplacental ratio results in terms of the well-being of the newborn and need for hospitalization in the intensive care unit, larger series of study groups are needed.

Keywords: Cerebroplacental ratio, middle cerebral artery, umbilical artery.

\section{Giriş}

2013 yılında American College of Obstetrician and Gynecologist (ACOG), term gebelik tanımlamasında yeni bir düzenleme yapmış ve $37^{+0}$ ile $38^{+6}$ hafta arasını erken-term, $39^{+0}$ ile $40^{+6}$ hafta arasını full-term, $41^{+0}$ ile $41^{+6}$ hafta arasını geç-term, $42^{+0}$ hafta ve üzerini ise postterm olarak belirlemiştir (1). Birçok çalışma, geçterm ve post-term gebeliklerin perinatal morbidite ve mortalite riskini arttırdığını göstermiştir $(2,3)$. $\mathrm{Bu}$ çalışmalarda gösterilen başlıca morbidite ve mortalite nedenleri gestasyonel hipertansiyon, baş-pelvis uygunsuzluğu ile seyreden uzamış doğum eylemi, doğum yaralanmaları ve hipoksik iskemik ensefalopatidir. Moster ve arkadaşları, 2010 yılında yaptıkları çalışmada post-term doğumlarda serebral palsi oranını artmış olarak bulmuşlardır (4).

Serebroplasental oran (cerebroplacental ratio, CPR), orta serebral arter pulsatilite indeksi (MCA$\mathrm{PI})$ ve umbilikal arter pulsatilite indeksi (UA-PI) arasındaki orandır (5). CPR 1.0'den küçükse anormal olarak kabul edilir (6). CPR'nin olumsuz gebelik sonuçları (7), intrapartum fetal distres (8), yenidoğan kordon kan gazı $\mathrm{pH}$ değeri düşüklüğü (9) ve yenidoğan yoğun bakım ünitesine (YDYBÜ) yatış (10) oranlarında artış ile ilişkili olduğunu gösteren yeni yayınlar mevcuttur.

Bu çalışmanın amacı, 41 hafta ve üzeri komplike olmayan geç-term ve post-term gebeliklerde fetal Doppler akım özelliklerini karşılaştırmak ve bunların doğum sonrası sonuçlara etkisini değerlendirmektir.

\section{Gereç ve Yöntem}

$\mathrm{Bu}$ çalışma 17832 sayı ve 18.08.2017 tarihli Üniversite Etik Kurul onayını takiben, 01.07.2017
- 01.10.2017 tarihleri arasında, T.C. Sağlık Bakanlığı Sağlık Bilimleri Üniversitesi Ümraniye Eğitim ve Araştırma Hastanesi Kadın Hastalıkları ve Doğum Polikliniği'ne başvuran, gebelik haftası $41^{+0}$ ve üzerinde olan, düşük risk grubundaki (gestasyonel hipertansiyon, preeklampsi, gestasyonel diabet, fetal anomali, fetal gelişim kısıtııı̆ı gibi maternal ve fetal komplikasyonları olmayan) tekil canlı gebeliği olan 98 hastayla prospektif olarak yapıldı. $41^{+0}-41^{+6}$ hafta arasındaki 67 hasta geç-term grubu, $42^{+0}$ hafta ve üzerindeki 31 hasta post-term grubu oluşturdu. Tüm hastaların gebelik haftası son adet tarihi ve erken dönem sonografi verileri doğrulanarak hesaplandı. Hastaların yaş, boy, kilo, gravide, parite, post-term doğum öyküsü, hastaneye yatışından doğuma kadar geçen süre, doğum şekli, mekonyumlu amniotik sıvı ve fetal distres varlığı kayıt altına alındı. Geç-term veya postterm gebelik endikasyonuyla, doğum şekli ve zamanlaması açısından perinatoloji polikliniğine konsülte edilen hastalara gri skala ve renkli Doppler sonografik incelemesi Toshiba Aplio 500 (Toshiba Medical Systems, Japan) ile perinatoloji uzmanı eşliğinde yapıldı. Ölçümler, hasta supin pozisyonda iken, transabdominal olarak yapıldı. Doppler incelemeleri sırasında fetal solunum ve hareketlerin olmamasına dikkat edildi. Umbilikal kordonun serbest yüzen kısımlarından, orta serebral arter 0 dereceye yakın açıyla, çıkış yerinin hemen distalinden ve her iki uterin arter mesane boşken, uterin arterlerin serviks hizasında uterusa giriş yerlerinden Doppler ölçümleri yapıldı. Rutin fetal biyometri ölçümleri, fetal kilo tahmini (Hadlock I), amniyotik sıvı hacmi (en derin cep, $\mathrm{mm}$ ) biyofizik profil ve umbilikal arter pulsatilite indeksi, umbilikal arter rezistans 
indeksi (UA-RI), orta serebral arter pulsatilite indeksi, orta serebral arter rezistans indeksi (MCA-RI), ortalama uterin arter pulsatilite indeksi (UtA-PI) (sağ ve sol uterin arter ortalaması) değerleri kaydedildi. Serebroplasental oran (CPR) hesaplandı; 1.0 ve üstündeki değerler normal olarak kabul edildi. NST ve Bishop skoru sonucuna göre oksitosin ya da prostaglandin E2 ile doğum eylemi indüksiyonuna başlandı. Yenidoğan kordon kan gazı $\mathrm{pH}$ değeri, doğum kilosu, 5. dakika Apgar skoru, yenidoğan yoğun bakım ihtiyacı kayda alındı.

\section{Istatistiksel Analiz}

Tüm veriler Statistical Package for the Social Science (SPSS) version 21 (Chicago, IL) veri tabanına kaydedildi. Verilerin normal dağılıma uygunluğu Kolmogorov-Smirnov testi ile analiz edildi. Normal dağılım gösteren parametrik verilerin karşılaştırılmasında Student's $t$ testi, normal dağılım gösteren kategorik verilerin karşılaştııımasında Ki-kare testi kullanıldı. $p<0,05$ değeri istatistiksel olarak anlamlı kabul edildi.

\section{Bulgular}

Çalışmamıza 98 hasta dahil edildi. $41^{+0}-41^{+6}$ hafta arasındaki 67 hastadan oluşan geç-term grubu ile $42^{+0}$ hafta ve üzerindeki 31 hastadan oluşan post-term grubu karşılaştırıldığında, iki grup arasında ortalama maternal yaş $(p=0,28)$, gravidite $(p=0,94)$, nulliparite oranı $(p=0,79)$, ortalama vücut kitle indeksi $(p=0,33)$, postmatür doğum öyküsü oranı $(p=0,75)$ açısından istatistiksel olarak fark görülmedi (Tablo-1). Her iki grubun doğum eylemi indüksiyonunun başlangıcından doğuma kadar geçen ortalama süre $(p=0,803)$, amniyotik sıvı indeksi $(p=0,24)$, amniyotik sıvıda mekonyum varlığı $(p=0,67)$, fetal distres oranı $(p=0,14)$ ve sezaryen oranı $(p=0,24)$ açısından anlamlı fark gözlenmedi. Geç-term grupta en sık sezaryen nedeni ilerlemeyen eylem iken, post-term olgularda en sık neden fetal distres olarak görüldü.

Tablo-1. Olguların demografik verilerinin, obstetrik özelliklerinin, doğum şekillerinin ve yenidoğanların klinik özelliklerinin gruplar arasında karşılaştırılması.

\begin{tabular}{lccc}
\hline & $\begin{array}{c}\text { Geç-term olgular } \\
(\mathbf{n}=\mathbf{6 7})\end{array}$ & $\begin{array}{c}\text { Post-term olgular } \\
(\mathbf{n}=\mathbf{3 1})\end{array}$ & p değeri \\
\hline Maternal yaş (yıl) & $27,01 \pm 4,2$ & $25,9 \pm 4,4$ & 0,28 \\
Gravidite & $2,06 \pm 1,2$ & $2,08 \pm 0,8$ & 0,94 \\
Nulliparite [oran (\%)] & $23 / 67(34,3)$ & $10 / 21(32,2)$ & 0,79 \\
Vücut Kitle İndeksi (kg/m²) & $26,9 \pm 2,6$ & $27,1 \pm 3,1$ & 0,33 \\
Gebelik haftası (hafta) & $41,1 \pm 0,9$ & $42,1 \pm 0,3$ & $<0,001$ \\
Doğuma kadar geçen süre (saat) & $23,5 \pm 14,9$ & $22,7 \pm 14,8$ & 0,80 \\
Amniotik sıvı indeksi (en derin cep, mm) & $32,5 \pm 11,5$ & $37,1 \pm 18,06$ & 0,24 \\
Oligohidramnios [oran (\%)] & $18 / 67(26,8)$ & $13 / 31(41,9)$ & 0,14 \\
Mekonyum varlığı [oran (\%)] & $23 / 67(34,3)$ & $12 / 31(38,7)$ & 0,67 \\
Fetal distres [oran (\%)] & $6 / 67(8,9)$ & $6 / 31(19,3)$ & 0,14 \\
Vajinal doğum [oran (\%)] & $53 / 67(79,1)$ & $21 / 31(67,7)$ & 0,22 \\
Sezeryan [oran (\%)] & $14 / 67(20,9)$ & $10 / 31(32,3)$ & 0,24 \\
Doğum kilosu (gr) & $3500 \pm 358$ & $3693 \pm 461$ & $\mathbf{0 , 0 4 5}$ \\
4000 g üstü yenidoğan [oran (\%)] & $6 / 67(8,9)$ & $10 / 31(32,2)$ & $\mathbf{0 , 0 0 4}$ \\
5. dakika Apgar skoru & $8,8 \pm 1,2$ & $9,1 \pm 0,8$ & 0,264 \\
Kordon kan gazı arter pH değeri & $7,34 \pm 0,07$ & $7,33 \pm 0,1$ & 0,490 \\
YDYBÜ intiyacı [oran (\%)] & $12 / 67(17,9)$ & $12 / 31(38,7)$ & $\mathbf{0 , 0 2 6}$ \\
\hline
\end{tabular}

YDYBÜ: Yenidoğan Yoğun Bakım Ünitesi 
Ortalama doğum kilosu geç-term olgularda $3500 \pm 358$ gr iken post-term olgularda $3693 \pm 461$ gr olup, bu bulgu istatistiksel olarak anlamlı bulunmuştur $(p=0,045)$. Geç-term grupta altı yenidoğanın $(\% 8,9)$ tartısı $4000 \mathrm{~g}$ üzerindeyken post-term olgularda bu sayı $10(\% 32,2)$ olarak bulunmuştur $(p=0,004)$.

Her iki grubun 5. dakika Apgar skoru $(p=0.264)$ ve kordon kan gazı $\mathrm{pH}$ değerleri $(p=0,94)$ arasında istatistiksel olarak anlamlı farklılık yoktur. Ancak yenidoğan yoğun bakım ünitesine yatış oranları geç-term grupta \%17,9, post-term grupta ise $\% 38,7$ olarak bulundu $(p=0,026)$. Yenidoğan yoğun bakım yatış ünitesine en sık yatış nedenleri yenidoğanın geçici solunum sıkıntısı ve sarılıktı.

Olguların Doppler akım özellikleri karşılaştıııldığında (Tablo-2), her iki grubun ortalama UtA-PI değerleri arasında fark gösterilememiştir $(p=0,112)$. Ortalama UA PI değeri gruplar arasında farklılık göstermezken $(p=0,81)$, post-term grupta ortalama UA-RI değeri
$(0,51 \pm 0,04)$ anlamlı olarak daha düşük bulunmuştur $(p=0,02)$.

Ortalama MCA-RI değerleri arasında fark gözlenmezken $(p=0,148)$, ortalama MCA-PI değeri geç-term grupta $1,3 \pm 0,5$ iken post-term grupta $1,1 \pm 0,2$ 'dir ve bu farklılık istatistiksel olarak anlamlı bulunmuştur $(p=0,046)$. UA-PI / MCA-PI değeri olarak hesaplanan CPR, geç-term grupta daha düşük olsa da $(1,4 \pm 0,4)$ bu farklılık istatistiksel olarak anlamlı değildir $(p=0,126)$ ve tanımlanan kritik eşik değer olan 1,0 oranından uzak bulunmuştur.

Çalışmaya dahil edilen olgulardan fetal distres nedeniyle acil sezaryene ve sonrasında yoğun bakım ünitesine alınanlar, kordon kan gazı $\mathrm{Ph}$ değeri 7,1 altında olanlar ve 5. dakika APGAR skoru $<7$ olan hastalar perinatal morbiditesi olan grup olarak tanımlandı ve perinatal morbiditesi olan ve olmayan grubun iki grubun Doppler akım özelliklerini karşıllaştırıldı (Tablo-3). Her iki gruptaki veriler arasında anlamlı fark gösterilememiştir.

Tablo-2. Olguların Doppler akım özelliklerinin karşılaştırılması.

\section{Geç-term Olgular $(n=67)$}

$\begin{array}{lc}\text { UtA-PI } & 1,06 \pm 0,37 \\ \text { UA-PI } & 0,83 \pm 0,21 \\ \text { UA-RI } & 0,55 \pm 0,07 \\ \text { MCA-PI } & 1,3 \pm 0,5 \\ \text { MCA-RI } & 0,69 \pm 0,11\end{array}$

Post-term Olgular $(\mathbf{n}=\mathbf{3 1})$

p değeri

$\begin{array}{cc}0,97 \pm 0,1 & 0,11 \\ 0,82 \pm 0,27 & 0,81 \\ 0,51 \pm 0,04 & \mathbf{0 , 0 2} \\ 1,1 \pm 0,2 & \mathbf{0 , 0 4} \\ 0,65 \pm 0,1 & 0,15 \\ 1,4 \pm 0,4 & 0,13\end{array}$

UtA: Uterin arter, UA: Umbilikal arter, MCA: Orta serebral arter, PI: Pulsatilite indeksi, RI: Rezistans indeksi, CPR: Serebroplasental oran

Tablo-3. Perinatal morbiditesi olan olgularla olmayan olguların Doppler akım özelliklerinin karşılaştırılması.

\begin{tabular}{lccc}
\hline & $\begin{array}{c}\text { Perinatal Morbiditesi Olmayanlar } \\
(\mathbf{n}=\mathbf{8 2})\end{array}$ & $\begin{array}{c}\text { Perinatal Morbiditesi Olanlar } \\
(\mathbf{n}=\mathbf{1 6})\end{array}$ & p değeri \\
\hline UtA-PI & $0,95 \pm 0,10$ & $1,08 \pm 0,32$ & 0,11 \\
UA-PI & $0,81 \pm 0,20$ & $0,88 \pm 0,32$ & 0,42 \\
UA-RI & $0,54 \pm 0,07$ & $0,53 \pm 0,08$ & 0,68 \\
MCA-PI & $1,25 \pm 0,45$ & $1,13 \pm 0,36$ & 0,29 \\
MCA-RI & $0,68 \pm 0,10$ & $0,67 \pm 0,11$ & 0,66 \\
CPR & $1,54 \pm 0,5$ & $1,37 \pm 0,6$ & 0,31 \\
\hline
\end{tabular}

UtA: Uterin arter, UA: Umbilikal arter, MCA: Orta serebral arter, PI: Pulsatilite indeksi, RI: Rezistans indeksi, CPR: Serebroplasental oran 


\section{Tartışma}

Post-term gebeliklerdeki Doppler akım değişikliklerini araştıran az sayıda çalışma vardır. Gün aşımı gebeliklerde ilerleyici olarak artan plasental yetmezlik bulgularını Doppler verileriyle ortaya koymaya çalışan bu çalışmaların verileri de birbirleriyle tutarsızlık göstermektedir. Bazı çalışmalar, olumsuz perinatal sonuçlarla ilişkili post-term gebeliklerde fetal serebral dolaşımda redistribüsyon olduğunu, beyin koruyucu benzeri bir etkiyle oligohidramniosun meydana geldiğini göstermiştir (11). Ancak bu durumun aksine, post- term gebeliklerde oligohidramnios varlığından bağımsız, Doppler indekslerinde anlamlı bir değişiklik olmadığını gösteren çalışmalar da vardır $(12,13)$. Lam ve arkadaşları, 41 hafta üzerindeki, risk faktörü olmayan gebelerin fetal serebro-plasental Doppler indeksleriyle amniotik sıvı miktarı arasındaki ilişkiyi değerlendiren prospektif gözlemsel bir çalışma yayınlamışlardır (12). Bu çalışma doğum eylemi sırasında koyu mekonyumla boyalı amniotik sıvı olasılığıyla MCA-PI değerlerinin ters ilişkili olduğunu göstermiştir. Aynı çalışmada oligohidramnios veya mekonyumla boyalı amniotik sIVI izlenen olgularda hem UA-PI değerlerinin hem de CPR'nin anlamlı farklılık göstermediği ortaya konulmuştur. Lebovitz ve arkadaşları 40 haftanın üzerinde, düşük risk grubundaki 120 hastanın uterin, umbilikal, orta serebral arter Doppler indeksleriyle, serebroplasental oranlarını prospektif olarak değerlendirmiştir (13). Bu grup içerisinde olumsuz perinatal sonuçları olan hastaların verilerinin diğer gruptan farklı olmadığı gösterilmiştir. El-Sokkary ve arkadaşları, CPR'nin $<0,85$ olduğu gün aşımı gebeliklerde fetal distres olasılığının arttığını göstermiştir (14). Daha güncel çalışmalardan D'Antonio ve arkadaşlarının çalışmasında, 41 hafta üzerindeki gebe grubunda CPR ile olumsuz perinatal sonuçlar arasında bir ilişki olmadığı gösterilmiştir (15). Son olarak Kauppinen ve arkadaşları, 41 hafta üzerindeki hastalarda olumsuz perinatal sonuçları olan ve olmayan iki grupta uterin, umbilikal, orta serebral arter ve CPR oranlarını karşılaştırmıştır (16). Her iki grupta Doppler verileri açısından fark gösterilememiştir.

Her ne kadar riskli gebeliklerde Doppler indekslerinin karşılaştırıldığı çok sayıda çalışma bulunsa da sadece post-term gebelik grubunda yapılmış kısıtlı sayıda çalışma bulunmaktadır. Çalışmamız geç-term hasta grubu ile post-term hasta grubunun Doppler verilerini karşılaştıran ilk çalışma olarak dikkati çekmektedir. Çalışmanın güçlü yanları arasında prospektif bir çalışma olması, tek bir merkezden yapılmış olması, ultrasonografi incelemelerinin aynı kişi tarafından yapılmış olması gibi faktörler sayılabilir. Postterm olgularda UA-PI ve MCA-PI değeri anlamlı olarak düşük bulunmasına rağmen CPR değerleri açısından fark gösterilememiştir.

\section{Sonuç}

Çalışma grubumuz, olumsuz perinatal sonuçları olan ve olmayanlar olarak karşılaştırıldığında, hiçbir Doppler verisi açısından anlamlı farklııı gösterilememiştir. Ancak, geç-term ve post-term gebeliklerde, Doppler bulgularında kötü perinatal sonuçları da öngörecek, anlamlı farklılıkları ortaya koyacak daha geniş gruplarla yapılacak çalışmalara intiyaç bulunmaktadır.

\section{Çıkar çatışması}

Yazarlar çıkar çatışması beyan etmemişlerdir.

\section{Kaynaklar}

1. ACOG Committee Opinion No: 579. Definition of term pregnancy. Obstet Gynecol 2013; 122 (5): $1139-40$.

2. Cheng YW, Nicholson JM, Nakagawa S, Bruckner TA, Washington AE, Caughey AB. Perinatal outcomes in low-risk term pregnancies: do they differ by week of gestation? Am J Obstet Gynecol 2008; 199 (4): $370 . e 1-7$.

3. MacDorman MF, Kirmeyer S. Fetal and perinatal mortality, United States, 2005. Natl Vital Stat Rep $2009 ; 57$ (8): 1-19.

4. Moster D, Wilcox AJ, Vollset SE, Markestad T, Lie RT. Cerebral palsy among term and post term births. JAMA 2010; 304 (9): 976-82.

5. Bhide A, Badade A, Khatal K. Assessment of reproducibility and repeatability of cerebro-placental ratio. Eur J Obstet Reprod Biol 2019; 235:106-9.

6. Alfirevic Z, Stampalija T, Dowswell T. Fetal and umbilical Doppler ultrasound in high-risk pregnancies. Cochrane Database Syst Rev 2017; 6: CD007529. 
7. Khalil A, Morales-Rosello $\mathrm{J}$, Khan $\mathrm{N}$, et al. Is cerebroplacental ratio a marker of impaired fetal growth velocity and adverse pregnancy outcome? Am J Obstet Gynecol 2017; 216 (6): 606.e1-10.

8. Dall'Asta A, Ghi T, Rizzo G, et al. Cerebroplacental ratio assessment in early labor in uncomplicated term pregnancy and prediction of adverse perinatal outcome: prospective multicenter study. Ultrasound Obstet Gynecol 2019; 53 (4): 481-7.

9. Morales-Rosello J, Khalil A, Morlando M, Bhide A, Papageorghiou A, Thilaganathan B. Poor neonatal acidbase status in term fetuses with low cerebroplacental ratio. Ultrasound Obstet Gynecol 2015; 45 (2): 156-61.

10. Khalil A.A, Morales-Rosello J, Morlando M, et al. Is fetal cerebroplacental ratio an independent predictor of intrapartum fetal compromise and neonatal unit admission? Am J Obstet Gynecol 2015; 213: 54.e1-10.

11. Oz AU, Holub B, Mendilcioglu I, Mari G, Bahado-Singh RO. Renal artery Doppler investigation of the etiology of oligohydramnios in post term pregnancy. Obstet Gynecol 2002; 100 (4): 715-8.

12. Lam H, Leung WC, Lee CP, Lao TT. The use of fetal Doppler cerebroplacental blood flow and amniotic fluid volume measurement in the surveillance of postdated pregnancies. Acta Obstet Gynecol Scand 2005 Sep;84 (9): 844-8.

13. Lebovitz O, Barzilay E, Mazaki-Tovi S, Gat I, Achiron R, Gillboa Y. The clinical value of maternal and fetal Doppler parameters in low-risk postdates pregnancies - a prospective study. J Matern Fetal Neonatal Med 2018 Nov; 31 (21): 2893-7.

14. El-Sokkary M, Omran M, Ahmed H. Ratio of middle cerebral artery/umbilical artery Doppler velocimetry and status of newborn in postterm pregnancy. J Am Sci 2011; 7 (4): 542-9.

15. D'Antonio F, Patel D, Chandrasekharan N, Thilaganathan B, Bhide A. Role of cerebroplacental ratio for fetal assessment in prolonged pregnancy. Ultrasound Obstet Gynecol 2013; 42 (2): 196-200.

16. Kauppinen T, Kantomaa T, Tekay A, Mäkikallio K. Placental and fetal hemodynamics in prolonged pregnancies. Prenat Diagn 2016; 36 (7): 622-7. 